\title{
Periodic pattern formation in reaction-diffusion systems: An introduction for numerical simulation
}

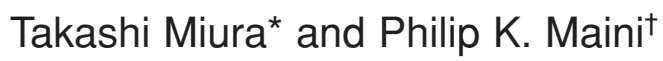 \\ * Department of Human Anatomy and Genetics and \\ + Centre for Mathematical Biology, Mathematical Institute, University of Oxford, UK
}

\begin{abstract}
The aim of the present review is to provide a comprehensive explanation of Turing reaction-diffusion systems in sufficient detail to allow readers to perform numerical calculations themselves. The reaction-diffusion model is widely studied in the field of mathematical biology, serves as a powerful paradigm model for selforganization and is beginning to be applied to actual experimental systems in developmental biology. Despite the increase in current interest, the model is not well understood among experimental biologists, partly because appropriate introductory texts are lacking. In the present review, we provide a detailed description of the definition of the Turing reaction-diffusion model that is comprehensible without a special mathematical background, then illustrate a method for reproducing numerical calculations with Microsoft Excel. We then show some examples of the patterns generated by the model. Finally, we discuss future prospects for the interdisciplinary field of research involving mathematical approaches in developmental biology.
\end{abstract}

Key words: mathematical modeling, numerical simulation, pattern formation, Turing.

\section{Introduction: Periodic pattern formation in biological systems and the Turing reaction-diffusion model}

It has been reported that, in many cases during development, periodic patterns emerge. Examples include the skin pigment pattern in zebra (Bard, 1981; Murray, 2003), angelfish (Kondo \& Asai, 1995; Shoji et al., 2003), zebrafish (Asai et al., 1999) and sea shells (Meinhardt, 1995), feather follicle formation (Jung et al., 1998), tooth development (SalazarCiudad \& Jernvall, 2002) and digit formation during limb development (Newman \& Frisch, 1979; Maini \& Solursh, 1991; Dowine and Newman, 1994, 1995; Miura and Shiota, 2000a, 2000b; Miura et al., 2000; Moftah et al., 2002). Certain aspects of these pattern formation processes cannot be easily explained simply by the combination of morphogen gradients.

Recently, some developmental biologists have started using the Turing reaction-diffusion model for this type of pattern formation. This model was originally proposed by the British mathematician Alan Turing (Turing, 1952) and a significant amount of

Correspondence: Takashi Miura, Department of Anatomy and Developmental Biology, Kyoto University Graduate School of Medicine, Yoshida Konoe-Chou, Sakyo-ku, 606-8501, Japan. Email: miura-takashi@umin.ac.jp

This paper is based on the winning article of the 2000 Incentive Prize of the Japanese Association of Anatomists.

Received 8 March 2004; accepted 14 April 2004. work has been done using this idea in the field of mathematical biology (for reviews, see Bard, 1990; Meinhardt, 1995; Murray, 2003). This model hypothesizes the existence of two molecules, an activator and an inhibitor, and, if they interact with each other in a specific manner (see below), a periodic pattern is formed from a homogeneous initial spatial distribution of activator and inhibitor. The qualitative explanation of pattern formation is as follows: because the activator has an ability to enhance its own production, any small peak of activator in the initial distribution is amplified. As the activator peaks grow, inhibitor peaks should also grow in response because the activator promotes the production of inhibitor. Inhibitor peaks should be less steep than activator peaks owing to the assumption that the inhibitor has a larger diffusion coefficient, which results in the inhibition of new activator peak formation near pre-existing peaks. This results in a periodic pattern of activator and inhibitor peaks (Meinhardt, 1995; Kondo, 2002).

This explanation itself has become well known to developmental biologists and some standard developmental biology textbooks have started to take up this topic (see Wolpert, 1998; Gilbert, 2000). Most experimental biologists can follow the description of the logic, but very few actually use the model because it is necessary to at least reproduce the numerical simulation results to do any serious scientific research using the model. One obstacle for this is that there is virtually no introductory review that is suitable for this purpose. Therefore, the aim of this 
text is to provide enough information for the reader to perform actual numerical calculations on reactiondiffusion models. We will provide an actual program written by Microsoft Excel (Redmond, WA, USA) and will provide a Mathematica program on request. The authors strongly encourage readers to read Murray (2003; Volume II, Chapter 2), which contains the basic mathematical explanation of why periodic patterns are formed in the model.

\section{Model equations}

\section{What is a differential equation?}

Most of the aforementioned texts that deal with reaction-diffusion systems are aimed at readers with a mathematical background, so they immediately plunge into the mantra-like string:

$$
\begin{aligned}
& u^{\prime}=f(u, v)+d_{u} \Delta u \\
& v^{\prime}=g(u, v)+d_{v} \Delta v
\end{aligned}
$$

which is very puzzling for most experimental biologists at first glance. These equations are called differential equations (more specifically, partial differential equations) and describe the rate of change of an internal state of a certain physical system in both time and space. To fully specify the problem requires three factors: (i) initial conditions; (ii) governing equations; and (iii) boundary conditions.

Initial conditions mean the values of the given system at the very beginning. The governing equations define the rules on how these values will change in time and space. Boundary conditions define how the system behaves at its boundary; for example, the system may be confined within a certain domain, so there would be no flux out of the boundaries. We will explain what these terms actually mean by using the simplest example. To facilitate understanding, the definitions of each variable are given in the Appendix.

\section{Initial conditions}

For simplicity, we will think about a quasi one-dimensional rod-like embryonic tissue (Fig. 1). We define horizontal length as 1 and vertical length as $d y(\ll 1$,

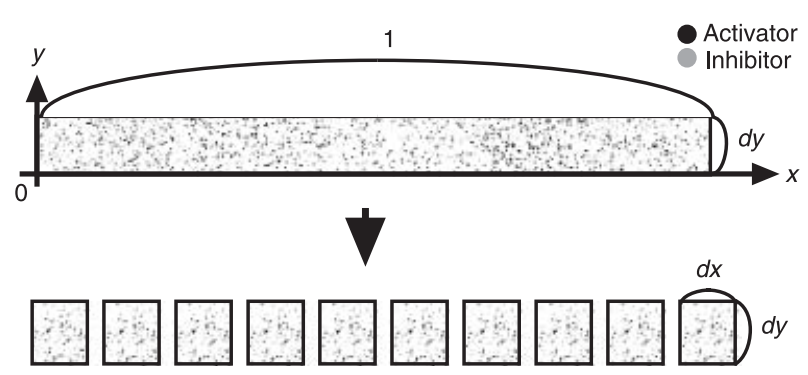

Figure 1. Definition of initial conditions and discretization in space. much smaller than 1) and suppose the distribution of molecules in the vertical direction is negligible. Cells inside this tissue produce two diffusible signaling molecules, the activator and the inhibitor, and these molecules control the production (or degradation) of both molecules and diffuse to neighboring cells. The distribution can be homogeneous or can have some prepattern, as shown in later sections, depending on the actual experimental situation.

\section{Discretization}

Because it is quite difficult to think directly about the time-course of spatial distribution of the molecules, at first we divide this rod-like structure into small pieces that have horizontal length $d x(d x \ll 1$, meaning $d x$ is much smaller than 1) and suppose the spatial distribution of activator and inhibitor in these small pieces to be homogeneous. Then, we will think about the concentration change of activator and inhibitor in these small elements. There are two factors that affect the concentration of activator and inhibitor in these small pieces: (i) the interaction of activator and inhibitor within each element; and (ii) the transfer of activator and inhibitor between each element and its two nearest neighbors.

We consider the sum of these two factors as the concentration change of these molecules within a specific element in a short period of time.

Basically, time should be continuous, but we consider updating the system in discrete time steps, $d t$. So, at time step $m$ (where $m$ is a positive integer), a time of $m \times d t$ has actually passed (see Fig. 2). We can define the concentration of activator molecule in the $n$th tissue element from the left-hand boundary at time $m \times d t$ as $p(n, m)$ and the concentration of inhibitor as $q(n, m)$.

\section{Reaction term}

At first we suppose the spatial distributions of activator and inhibitor at time $m \times d t$ are known and think about what will happen in each small tissue element after the short time period $d t$. Then, we will consider the events that occur solely inside each tissue piece. There are two types of Turing reaction-diffusion model that are known to generate spatial patterns: (i) the activatorinhibitor type; and (ii) the substrate-depletion type. In the activator-inhibitor type reaction-diffusion model (Fig. 3), the activator promotes its own production and promotes the production of inhibitor and the inhibitor inhibits the activator production and decays with time.

So, for example, if we set the rate of change of activator and inhibitor as $f(p, q)$ and $g(p, q)$, respectively, and consider:

$$
\begin{aligned}
& f(p, q)=0.6 p-q \\
& g(p, q)=1.5 p-2 q
\end{aligned}
$$




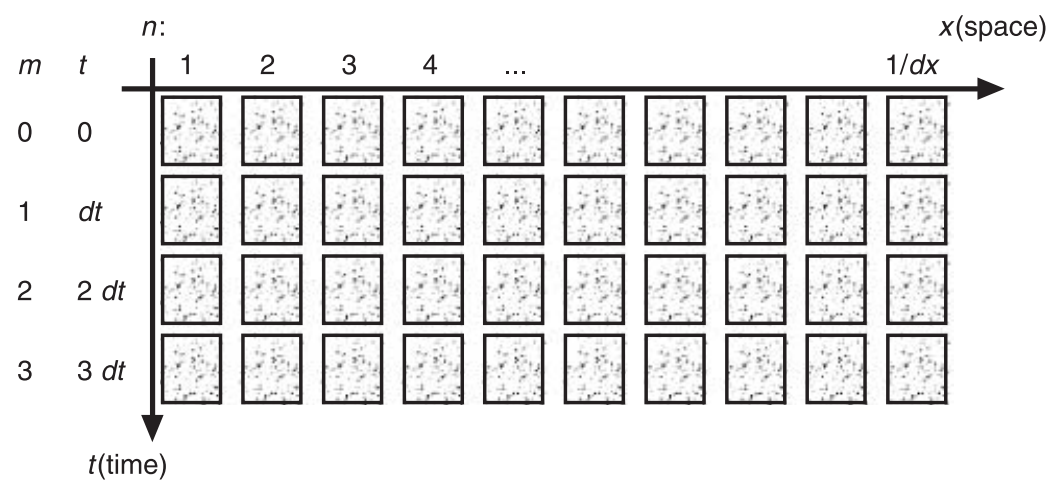

Figure 2. Discretization in space and time.

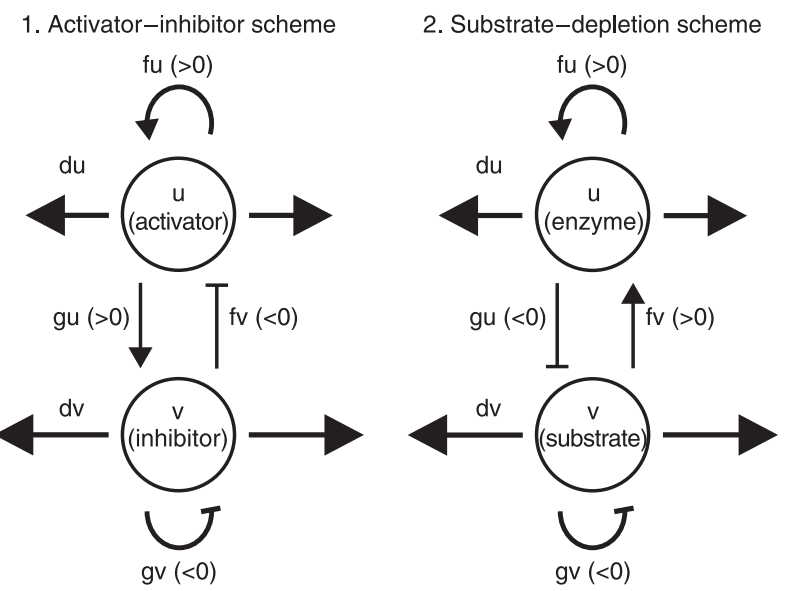

Figure 3. Schematic representation of the reaction term.

we then have a system in which $p$ is the activator and $q$ is the inhibitor. These are called the 'reaction terms'.

Actual biologically relevant functions will have saturation limits for the concentration of activator and inhibitor, so the reaction terms will have more complex form. For mathematical simplicity, we allow negative values for $p$ and $q$ and set their initial values to 0 . If we set actual concentrations of activator and inhibitor as $P$ and $Q$, they will then be transformed to $p$ and $q$, with $p=P-P_{0}$ and $q=Q-Q_{0}$, where $P_{0}$ and $Q_{0}$ are certain positive constants.

We can calculate the amount of change of activator and inhibitor during the time interval spanning $(m \times d t,(m+1) \times d t)$ as:

$$
\begin{aligned}
& f(p(m, n), q(m, n)) \times d t \\
& g(p(m, n), q(m, n)) \times d t
\end{aligned}
$$

respectively. The $d t$ term arises from observing that the actual increase in chemical concentration is obtained by multiplying the net rate of production by time.

\section{Diffusion term}

Next, we will consider the interaction between a tissue element and its two nearest neighboring tissue

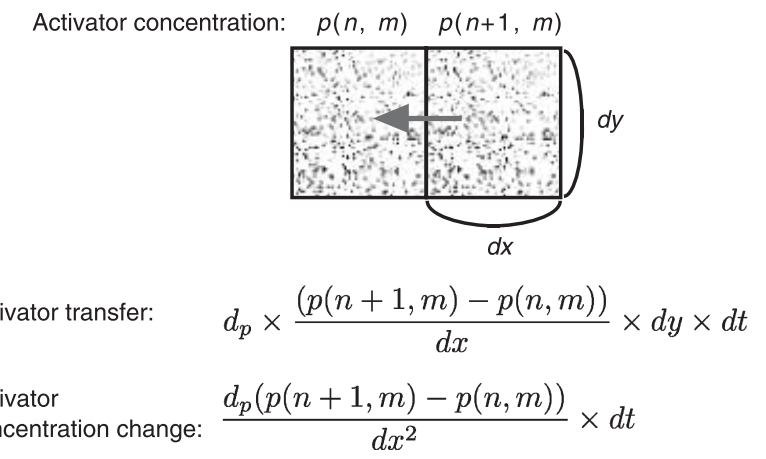

Figure 4. Transfer of activator or inhibitor between neighboring tissue pieces.

elements during the time interval $(m \times d t,(m+1) \times d t)$. In the biological context, there are many ways to transmit signals spatially but, for simplicity, here we consider that both activator and inhibitor diffuse passively between tissue elements.

If we suppose the concentration of activator in the $n$th tissue element is $p(n, m)$, then the concentration of activator in the tissue element to the right will be $p(n+1, m)$. In the case of simple diffusion, the amount of molecule transferred from element $n$ to $n+1$ is proportional to the concentration gradient $(p(n+1, m)-$ $p(n, m)) / d x$ and transverse length of the element $d y$ (Fick's Law). Therefore, the amount of activator that is transferred from the right neighboring piece (arrow in Fig. 4) is:

$$
d_{p} \times \frac{(p(n+1, m)-p(n, m))}{d x} \times d y \times d t
$$

where $d_{p}$ represents the diffusion coefficient of the activator. The concentration change induced by this transfer can be obtained by dividing this value by the area of the tissue element $(d x \times d y)$.

$$
\begin{gathered}
d_{p} \times \frac{(p(n+1, m)-p(n, m))}{d x} \times d y \times d t /(d x \times d y) \\
=\frac{d_{p}(p(n+1, m)-p(n, m))}{d x^{2}} \times d t
\end{gathered}
$$


Similarly, we can obtain the amount of activator transferred from the left neighboring element as:

$$
d_{p} \times \frac{(p(n-1, m)-p(n, m))}{d x} \times d y \times d t
$$

and the concentration change induced by the transfer as:

$$
d_{p} \times \frac{(p(n-1, m)-p(n, m))}{d x^{2}} \times d t
$$

Taken together, the change of activator concentration in element $n$ between time $m \times d t$ and $(m+1) \times d t$ is:

$$
d_{p} \times \frac{(p(n+1, m)+p(n-1, m)-2 \times p(n, m))}{d x^{2}} \times d t[9]
$$

Similarly, the concentration change of inhibitor is:

$$
d_{q} \times \frac{(q(n+1, m)+q(n-1, m)-2 \times q(n, m))}{d x^{2}} \times d t \quad[10
$$

in which $d_{q}$ is the diffusion coefficient of the inhibitor.

This part, which represents the effect of diffusion, is called the 'diffusion term' in the reaction-diffusion system.

\section{Governing equations}

Taking both reaction and diffusion into consideration, the changes of concentrations of activator and inhibitor during the time interval $(m \times d t,(m+1) \times d t)$ are:

$$
\begin{aligned}
& p(n, m+1)-p(n, m)=(f(p(n, m), q(n, m))+ \\
& \left.\quad d_{p}(p(n+1, m)+p(n-1, m)-2 \times p(n, m)) / d x^{2}\right) \times d t \\
& q(n, m+1)-q(n, m)=(g(p(n, m), q(n, m))+ \\
& \left.\quad d_{q}(q(n+1, m)+q(n-1, m)-2 \times q(n, m)) / d x^{2}\right) \times d t
\end{aligned}
$$

From these, we can calculate the concentrations of activator and inhibitor at time $(m+1) \times d t$ as:

$$
\begin{aligned}
& p(n, m+1)=p(n, m)+(f(p(n, m), q(n, m))+ \\
& \left.\quad d_{p}(p(n+1, m)+p(n-1, m)-2 \times p(n, m)) / d x^{2}\right) \times d t \\
& q(n, m+1)=q(n, m)+(g(p(n, m), q(n, m))+ \\
& \left.\quad d_{q}(q(n+1, m)+q(n-1, m)-2 \times q(n, m)) / d x^{2}\right) \times d t
\end{aligned}
$$

Therefore, if we know the initial spatial distribution of activator and inhibitor, we can obtain the concentration distribution of activator and inhibitor at arbitrary time by repeatedly applying this equation at each time step $d t$.

\section{Boundary conditions}

Not all tissue pieces obey the rule described above. In the left-most and right-most tissue we have to define special conditions. There are several ways to do this, depending on the biological situation. If the total number of tissue elements is $N_{\text {total, }}$, then $p(1, m)$ and
$p\left(N_{\text {total }}, m\right)$ have only one neighbor and we cannot treat them as above; that is, equations 12 only hold for integer $n$ with $n$ greater than 1 and less than $N_{\text {total. }}$.

This condition poses a constraint on the number of waves in a certain region. Specifically, the number of waves has to be an integer in the defined region. The effect of the boundary becomes stronger when there are only a small number of structures in the defined region.

\section{Periodic boundary condition}

We define the values of $p(1, m)$ and $p\left(N_{\text {total }}, m\right)$ to be equal for all $m$, so that the left-most tissue and right-most tissue are connected.

\section{Zero-flux boundary condition}

We assume that the boundary is impermeable; that is, no material is transferred across it. In this case, we have to define the change of $p(1, m)$ and $p\left(N_{\text {total }}, m\right)$ as follows:

$$
\begin{aligned}
& p(1, m+1)=p(1, m)+(f(p(1, m), q(1, m)) \\
& \left.\quad+d_{p}(p(2, m)-p(1, m)) / d x^{2}\right) \times d t \\
& p\left(N_{\text {total }}, m+1\right)=p(1, m)+\left(f\left(p\left(N_{\text {total }}, m\right), q\left(N_{\text {total }}, m\right)\right)\right. \\
& \left.\quad+d_{p}\left(p\left(N_{\text {total }}-1, m\right)-p\left(N_{\text {total }}, m\right)\right) / d x^{2}\right) \times d t
\end{aligned}
$$

This condition is also called the Newmann boundary condition.

\section{Fixed boundary condition}

In this case, we have to set $p(1, m)$ and $p\left(N_{\text {total }}, m\right)$ to specific values for all time. Hence:

$$
\begin{gathered}
p(1, m+1)=\alpha \\
p\left(N_{\text {total }}, m+1\right)=\beta
\end{gathered}
$$

for each iteration of the simulation where $\alpha$ and $\beta$ are fixed (non-negative) numbers. This is also called a Dirichlet condition.

\section{Transformation to continuous equation}

We can obtain the continuous differential equations corresponding to the discrete equations above by making $d t$ and $d x$ infinitely small. At first, we define the concentration of activator and inhibitor as $u(x, t)$ and $v(x, t)$, which are now continuous functions ( $x$ and $t$ are now real values instead of integers). Then, the above discrete governing equations become:

$$
\begin{gathered}
\frac{u(x, t+d t)-u(x, t)}{d t}=f(u(x, t), v(x, t))+ \\
d_{p} \frac{\frac{u(x+d x, t)-u(x, t)}{d x}-\frac{u(x, t)-u(x-d x, t)}{d x}}{d x} \\
\frac{v(x, t+d t)-v(x, t)}{d t}=g(u(x, t), v(x, t))+ \\
d_{a} \frac{\frac{v(x+d x, t)-v(x, t)}{d x}-\frac{v(x, t)-v(x-d x, t)}{d x}}{d x}
\end{gathered}
$$


If $d t$ and $d x$ tend to zero, the left-hand side becomes a first-order time differentiation and the right-hand side becomes a second-order spatial differentiation as follows:

$\partial u(x, t) / \partial t=f(u(x, t), v(x, t))+d_{p} \partial^{2} u(x, t) / \partial x^{2}$

$\partial v(x, t) / \partial t=g(u(x, t), v(x, t))+d_{q} \partial^{2} v(x, t) / \partial x^{2}$

These are the governing equations of a reactiondiffusion system.

Some readers may be puzzled to see the strange character ' $\partial$ '. This is a 'partial derivative' and is defined as a derivative of a function of several variables when all but one variable (the variable of interest) are held fixed during the differentiation. For example, the left-hand side of the equation deals with a rate of change with time of concentration at a fixed location in space, which means differentiating with $t$ while keeping $x$ fixed.

Moreover, if you remember the following conventions, you can decipher equations 1: (i) first-order time differentiation $\partial u / \partial t$ is sometimes written as $u^{\prime}$; (ii) firstorder space differentiation $\partial u / \partial x$ is sometimes written as $\nabla u$ (' $\nabla$ ' is called 'del' or 'Nabla'); (iii) second-order space differentiation $\partial^{2} u / \partial x^{2}$ is sometimes written as $\Delta u$ (' $\Delta$ ' is called Laplacian).

Equations 18 are based on the assumption that the domain is one-dimensional. The equations can be generalized to fully three-dimensional space, where they take the following form:

$$
\begin{aligned}
& \frac{\partial u}{\partial t}=f(u, v)+d_{p} \nabla^{2} u \\
& \frac{\partial v}{\partial t}=g(u, v)+d_{q} \nabla^{2} v
\end{aligned}
$$

The symbol $\nabla^{2} u$ is short-hand for $\left(\left(\partial^{2} / \partial x^{2}\right) u\right)+$ $\left(\left(\partial^{2} / \partial y^{2}\right) u\right)+\left(\left(\partial^{2} / \partial z^{2}\right) u\right)$, sometimes also written as $\Delta$. In this case, $u=u(x, y, z, t)$ and $v=v(x, y, z, t)$, where $(x, y, z)$ is position in three-dimensional space.

We describe the continuous equations only for deciphering equation 1, but actually an analytical treatment of the system is possible for the continuous case (see Murray, 2003). This is beyond the scope of the present paper so, from now on, we concentrate on the numerical calculation of the discrete version of the model.

\section{Numerical calculation of the reaction- diffusion system}

\section{Parameters and equations used in the numerical calculations}

The reaction-diffusion system we now focus on is as follows:

$$
\begin{aligned}
& \frac{\partial u}{\partial t}=0.6 u-v-u^{3}+0.0002 \frac{\partial^{2} u}{\partial x^{2}} \\
& \frac{\partial v}{\partial t}=1.5 u-2 v+0.01 \frac{\partial^{2} v}{\partial x^{2}}
\end{aligned}
$$

on spatial domain size $[0,1]$ with zero-flux boundary conditions.

\section{Numerical calculation in Microsoft Excel}

To construct the numerical calculation program, we use Microsoft Excel, which is one of the most widely available programs for biologists. As we will see below, it is too time consuming to undertake all the simulations with Excel, but it is worth trying at least once by yourself to get a better understanding of the numerical calculation and an appreciation of the pitfalls involved.

\section{Initial conditions}

Suppose we are using the system in equations 20 with domain size 1 and we discretize it with $d x=0.05$ and $d t=0.1$. In that case, we have 20 pieces of tissue $(1 / 0.05=20)$ that contain both activator and inhibitor, so we define the initial distribution of activator and inhibitor by assigning 20 random numbers to each tissue piece. To describe this in an Excel spreadsheet, we assume each column represents the concentration of activator or inhibitor in a specific tissue piece. So, the initial concentration of the activator and inhibitor can be expressed as a $20 \times 2$ matrix of numbers in the spreadsheet, as shown in Fig. 5. In the case below, we use cell B1-U2 to define the initial distribution of activator and inhibitor. This set of random numbers represents the small noise that should exist in the actual system and, as we will see later, any set of random numbers as the initial condition will eventually lead to a similar periodic pattern in this simulation.

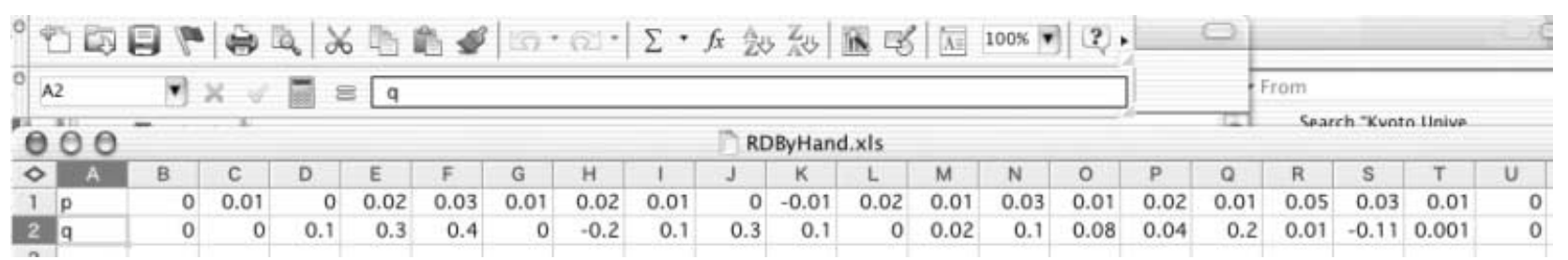

Figure 5. Initial distribution of $p$ and $q$ in the Excel spreadsheet. 


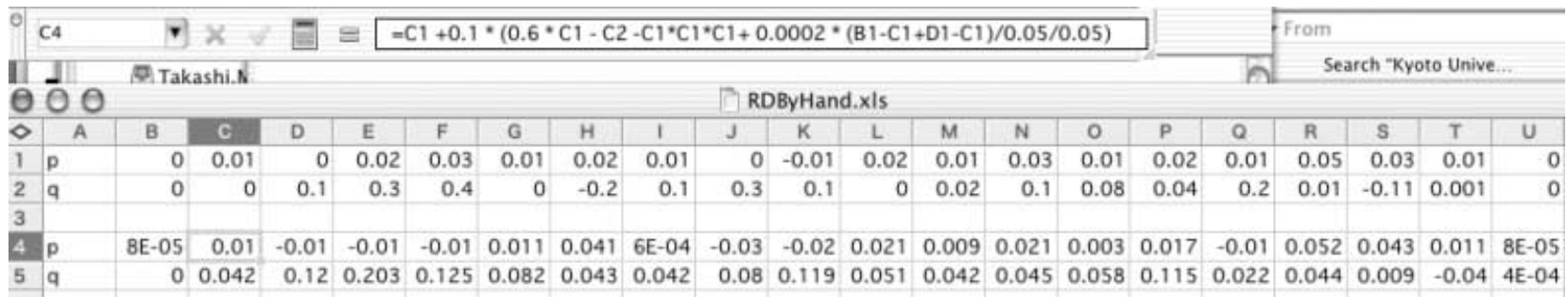

Figure 6. Entering governing equations of the reaction-diffusion system (equations 20) in the Excel spreadsheet.

\section{Governing equation}

First, we will calculate the concentration of activator $(p)$ and inhibitor $(q)$ at a certain tissue piece after time $d t$ has passed. For $d x=0.05$ and $d t=0.1$, the discrete version of the system is:

$$
\begin{aligned}
p(n, m+1)= & p(n, m)+(0.6 p(n, m)-q(n, m) \\
& -p(n, m)^{3}+0.0002(p(n+1, m) \\
& \left.+p(n-1, m)-2 \times p(n, m)) / 0.05^{2}\right) \\
& \times 0.01 \\
q(n, m+1)= & q(n, m)+(1.5 p(n, m)-2 q(n, m) \\
& +0.01(q(n+1, m)+q(n-1, m) \\
& \left.-2 \times q(n, m)) / 0.05^{2}\right) \times 0.01
\end{aligned}
$$

Let us calculate the activator and inhibitor concentrations for a certain column $(C)$ and write the values to $\mathrm{C} 4$ and $\mathrm{C} 5$. In this case, $p(n, m)=\mathrm{C} 1, p(n+1, m)$ $=\mathrm{D} 1, p(n-1, m)=\mathrm{B} 1, q(n, m)=\mathrm{C} 2, q(n+1, m)=\mathrm{D} 2$ and $q(n-1, m)=\mathrm{B} 2$. By substituting into equations 21, we can obtain the equation in cells C4 and C5:

$$
\begin{aligned}
\mathrm{C} 4: & =\mathrm{C} 1+(0.6 * \mathrm{C} 1-\mathrm{C} 2-\mathrm{C} 1 * \mathrm{C} 1 * \mathrm{C} 1 \\
& +0.0002 *(\mathrm{D} 1+\mathrm{B} 1-2 * \mathrm{C} 1) / 0.05 / 0.05) * 0.01 \\
\mathrm{C} 5: & =\mathrm{C} 2+(1.5 * \mathrm{C} 1-2 * \mathrm{C} 2+0.01 *(\mathrm{D} 2+\mathrm{B} 2 \\
& -2 * \mathrm{C} 2) / 0.05 / 0.05) * 0.01
\end{aligned}
$$

By evaluating these cells, we can obtain the concentration of activator and inhibitor in the tissue at column C. We can obtain values in other cells by applying the same equation (Fig. 6). Fortunately, Excel automatically converts these equations to appropriate forms by simply copying and pasting cells $\mathrm{C} 4$ and $\mathrm{C} 5$ to other cells.

\section{Boundary conditions}

As we have seen above, the tissues at the boundary should be treated separately. In this case, we use zero-flux conditions and define B4 and B5 as:

$$
\begin{aligned}
\mathrm{B} 4: & =\mathrm{B} 1+(0.6 * \mathrm{~B} 1-\mathrm{B} 2-\mathrm{B} 1 * \mathrm{~B} 1 * \mathrm{~B} 1 \\
& +0.0002 *(\mathrm{C} 1-\mathrm{B} 1) / 0.05 / 0.05) * 0.01 \\
\mathrm{~B} 5: & =\mathrm{B} 2+(1.5 * \mathrm{~B} 1-2 * \mathrm{~B} 2+0.01 *(\mathrm{C} 2 \\
& -\mathrm{B} 2) / 0.05 / 0.05) * 0.01
\end{aligned}
$$

and U4 and U5 as:

$$
\begin{aligned}
\text { U4: } & =\mathrm{U} 1+(0.6 * \mathrm{U} 1-\mathrm{B} 2-\mathrm{U} 1 * \mathrm{U} 1 * \mathrm{U} 1 \\
& +0.0002 *(\mathrm{~T} 1-\mathrm{U} 1) / 0.05 / 0.05) * 0.01 \\
\mathrm{U} 5: & =\mathrm{U} 2+(1.5 * \mathrm{U} 1-2 * \mathrm{U} 2+0.01 *(\mathrm{~T} 2 \\
& -\mathrm{U} 2) / 0.05 / 0.05) * 0.01
\end{aligned}
$$

By evaluating these cells, we can obtain the complete spatial distribution of activator and inhibitor at time $d t$.

\section{Numerical calculation}

To obtain the distribution of activator and inhibitor at a later time $t$, we have to carry out the process repeatedly (if $t=n d t$ we must iterate $n$ times). Fortunately Excel again automatically converts the equations to appropriate forms by simply copying and pasting the whole row to the rows below. By doing this 100-200 times repeatedly, you can observe that the concentrations gradually form a periodic structure, as shown in Fig. 7.

\section{Numerical calculation with Mathematica}

Obviously, it is too labor consuming to undertake the above procedure, so we generally use Mathematica (Wolfram Research, Champaign, IL, USA) to calculate the results. The result of the numerical calculation corresponding to the above example is shown in Fig. 8. You can see the emergence of a periodic structure from a nearly homogeneous initial state.

The details of the program can be provided electronically. (All the calculations in the present paper are performed by Mathematica and the source code (with additional instructions on linear stability analysis) is freely available on request from the authors.)

\section{Properties of Turing reaction-diffusion systems}

\section{Relationship between domain size and number of structures}

One characteristic of reaction-diffusion systems is that they tend to form structures of similar size (wavelength), so if the domain size is changed the number of structures should change, not the size of each structure. As can be seen in Fig. 9, a change in domain size results in a change in the number of structures. As can be seen in Murray (2003), the number of structures is dependent on the size of the whole domain. This property is reported considering the number of digits in a mutant mouse or chick, where it seems that larger limbs result in polydactyly, an increased number of digits. 


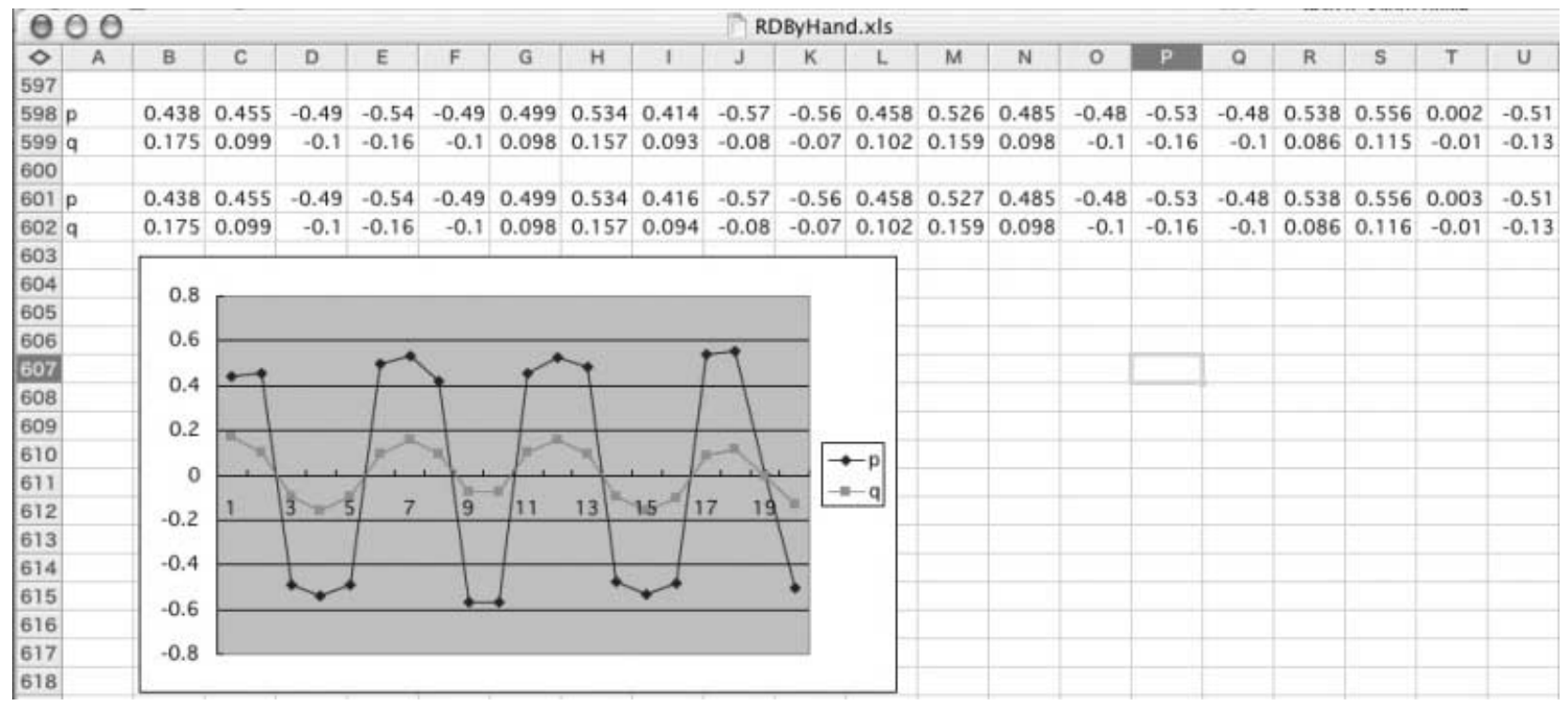

Figure 7. Numerical calculation results of the reaction-diffusion system (equations 20) by Excel.
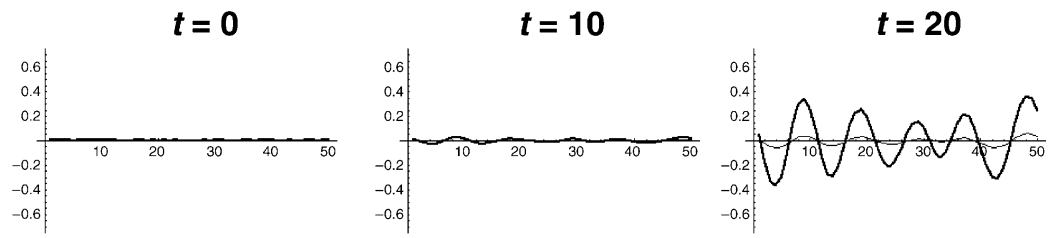

$\boldsymbol{t}=\mathbf{3 0}$

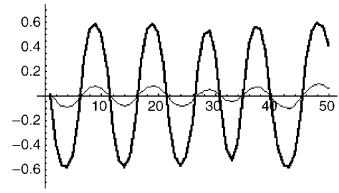

$t=40$
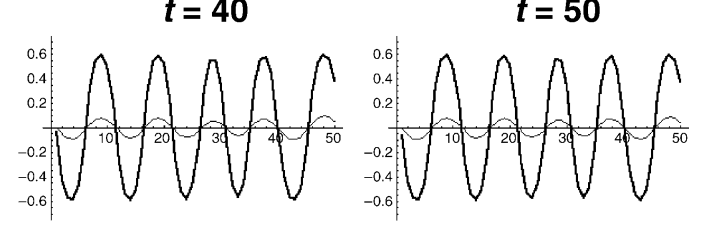

Figure 8. Time-course of numerical simulation of the reaction-diffusion model (equations 20). The thick line represents the distribution of the activator and the thin line represents the distribution of the inhibitor. See text for details.
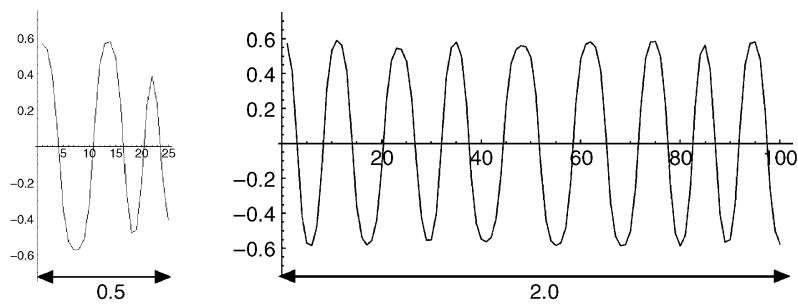

Figure 9. The numerical result of the reaction-diffusion model (equations 20) where the domain size is changed from 0.5 to 2.0. Distribution of the activator is depicted. Each structure stays the same size, but the number of structures increases with an increase in domain length.

\section{Changing initial conditions}

One characteristic of the Turing reaction-diffusion system is that it has the ability to form de novo stable periodic patterns; that is, without any prepattern. This is because the reaction-diffusion system forms patterns by amplifying specific wavelengths of the small minute fluctuations present in the initial conditions.

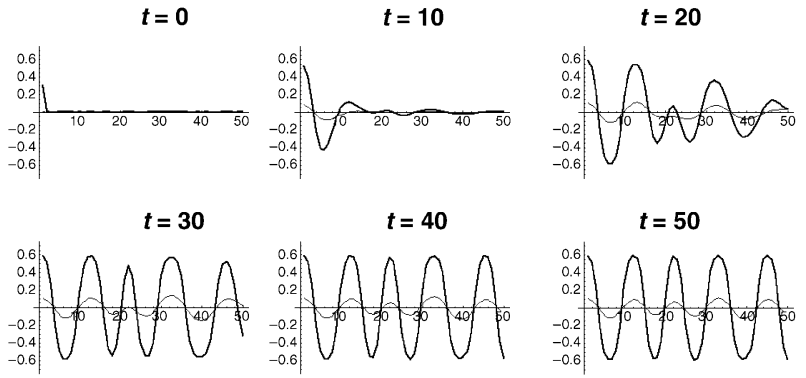

Figure 10. Numerical simulation of the reaction-diffusion model (equations 20) where the initial value of $p$ at the left-most point is increased.

(The detail of this explanation is described in Murray (2003).)

But what will happen if a prepattern does exist in this system? To see this, we change the initial value of $p$ at the left-most point, which corresponds to, for example, application of activator molecule to a certain embryonic tissue by using a bead. As you can see in Fig. 10, if the initial condition is not homogeneous, 

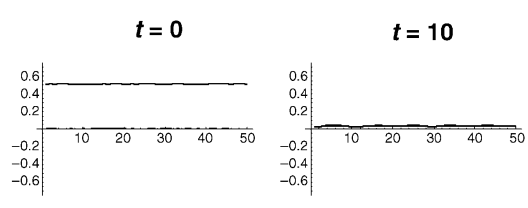

$t=40$
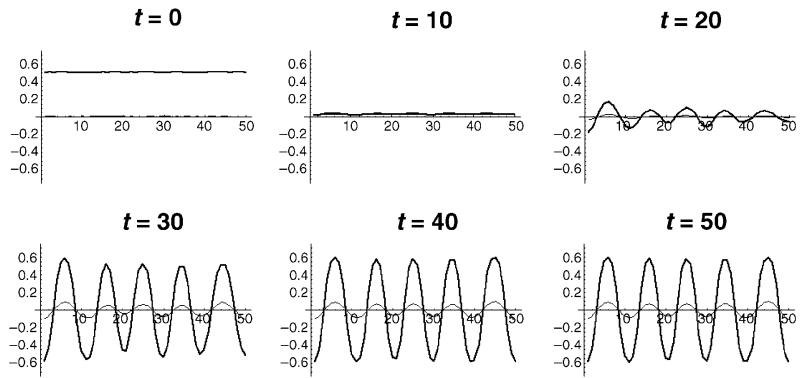

$t=50$

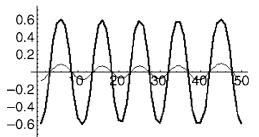

Figure 11. Numerical simulation of the reaction-diffusion model (equations 20) where the initial value of $p$ is increased uniformly. The thick line represents the distribution of the activator and the thin line represents the distribution of the inhibitor.

pattern formation can occur sequentially. However, the final periodic structure is more or less the same.

Even if we change the whole initial condition, the system quickly goes back to the uniform equilibrium value and then a stable periodic pattern begins to evolve in this case (Fig. 11). Moreover, it seems that it takes time to go back to the uniform equilibrium point and start the pattern formation process, so this suggests that adding activator molecule to the culture medium does not necessarily accelerate the pattern formation process. Detailed analysis on the pattern appearance speed has been recently undertaken in Miura and Maini (2004).

\section{Changing diffusion coefficients}

As you can see from the above discussion, the reaction-diffusion system has the ability to make a periodic pattern from an almost homogeneous initial state and the wavelength of the pattern is decided by the parameters in the reaction and diffusion terms. How is the wavelength of the pattern influenced if we change some of the parameters? Here, we deal with the simplest case, where the result can be predicted without detailed mathematical analysis.

At first, what will happen if the diffusion coefficients of both activator and inhibitor are decreased? This is experimentally assayed by Miura and Shiota (2000a) in a limb bud mesenchyme cell culture system. To implement this modification, we can multiply the diffusion coefficient by the scaling parameter, so the governing equations become:

$\partial u(x, t) / \partial t=f(u(x, t), v(x, t))+d_{p} \gamma \partial^{2} u(x, t) / \partial x^{2}$

$\partial v(x, t) / \partial t=g(u(x, t), v(x, t))+d_{q} \gamma \partial^{2} v(x, t) / \partial x^{2}$

where $0<\gamma<1$. Actually, the result is easy to understand without numerical calculation. If we set $\xi=x / \sqrt{\gamma}$, the above equation becomes:

$\partial u(\xi, t) / \partial t=f(u(\xi, t), v(\xi, t))+d_{p} \partial^{2} u(\xi, t) / \partial \xi^{2}$

$\partial v(\xi, t) / \partial t=g(u(\xi, t), v(\xi, t))+d_{q} \partial^{2} v(\xi, t) / \partial \xi^{2}$
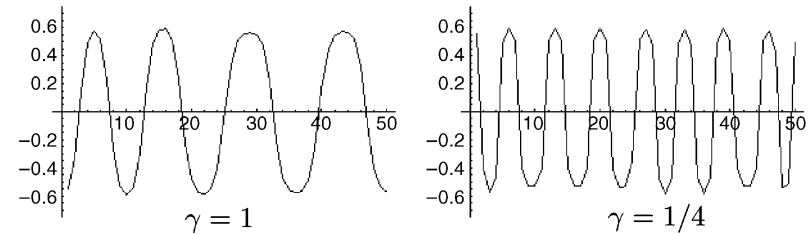

Figure 12. Numerical simulation of the reaction-diffusion model (equations 22) showing $p$ where $\gamma$ is changed from 1 to $1 / 4$.

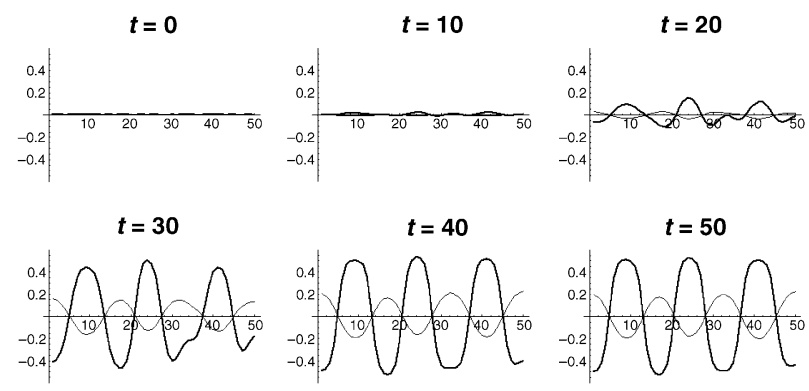

Figure 13. Numerical simulations of the reaction-diffusion model (equations 24). The thick line represents the distribution of $p$ and the thin line represents the distribution of $q$.

which is identical to the $\gamma=1$ case. Therefore, we are simply changing the spatial scale and, in this case, if we decrease $\gamma$, a greater number of structures should be formed in the domain. The result is confirmed by numerical calculation (Fig. 12).

\section{Cross-type reaction-diffusion model}

The activator-inhibitor scheme is well known among developmental biologists, but actually there is another type of reaction-diffusion model that has the ability to generate periodic pattern. This system, usually called the substrate-depletion system, also consists of two hypothetical molecules, the substrate and the enzyme. These molecules are hypothesized to interact in the following way (see Fig. 3): (i) substrate is consumed by the enzyme; and (ii) the enzyme produces itself by consuming substrate.

An example is:

$$
\begin{aligned}
& \frac{\partial u}{\partial t}=0.5 u+0.6 v-u^{3}+0.008 \frac{\partial^{2} u}{\partial x^{2}} \\
& \frac{\partial v}{\partial t}=-1.9 u-2 v+0.1 \frac{\partial^{2} v}{\partial x^{2}}
\end{aligned}
$$

where $u$ is the enzyme and $v$ is the substrate. Numerical simulations are shown in Fig. 13.

In the standard activator-inhibitor scheme, $u$ and $v$ must be in phase; $u$ peaks should be at the same place as $v$ peaks. However, in the above scheme (equations 24), the $u$ peaks are at $v$ valleys. Experimentally, this may be very important. For example, several studies suggest the molecular nature of the activator in digit formation during limb development (Dowine \& Newman, 1994; Miura \& Shiota, 2000b), but 

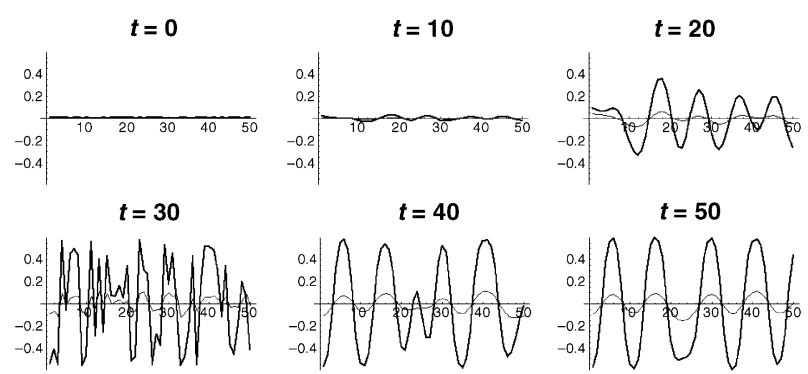

Figure 14. Numerical simulation of the reaction-diffusion model (equations 22). At $t=30$, the tissue is dissociated and then reaggregated. The disturbed pattern quickly reverts to normal. The thick line represents the distribution of the activator and the thin line represents the distribution of the inhibitor.

plausible candidates for the inhibitor have not been found. In the activator-inhibitor system, the inhibitor should be expressed at chondrogenic sites and have an ability to inhibit chondrogenesis. However, in the substrate-depletion system, the molecule that is expressed in the interdigital area and known to promote chondrogenesis also has an ability to generate pattern, which considerably increases the number of candidate molecules.

\section{Reaggregated tissue experiment}

As we saw in the previous subsection, one important characteristic of the Turing reaction-diffusion system is an ability to form a periodic pattern from various initial states and the process is quite robust. What will happen if the pattern is perturbed experimentally? Here, we try to emulate the most extreme case, where the tissue is dissociated into single cells and reaggregated. It has been shown that such a system can regenerate periodic structure in limb (Ros et al., 1994) and skin (Jiang et al., 1999).

The result is shown in Fig. 14. At $t=30$, tissue is dissociated into single pieces and randomly reaggregated again. This simulation shows that even if we severely perturb the pattern during the pattern formation process, the final periodic structure is more or less the same. This property is quite difficult to understand under the standard positional information hypothesis in which a graded morphogen profile is set up due to specialized structures forming a sourcesink system. However, the Turing-type reaction-diffusion model can easily reproduce and help explain the experimental result.

\section{Accuracy of the pattern formation mechanism}

Notice that the number of structures created by this system has a certain variance. If we perform numerical calculations with different random initial distributions, we can see a variation in the number of peaks. For example, in Fig. 15, the number of waves is approximately four, but you can observe a variation
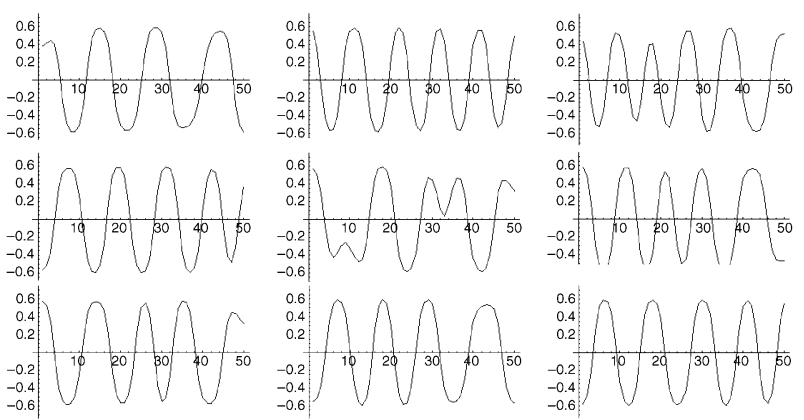

Figure 15. Numerical simulations of the reaction-diffusion model (equations 22). See text for details.
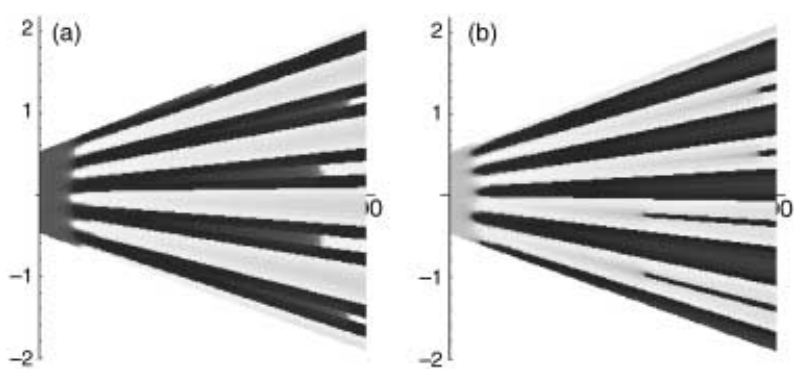

Figure 16. Numerical simulations of the reaction-diffusion model (equations 22) on growing domain. See text for details.

in the number of waves. Periodic structures in a biological system sometimes have variation in the number of elements (e.g. the number of hair follicles in the skin and the number of digits in the polydactylous mutant mice). It is difficult to explain this variance if we assume specific master genes exist that correspond to each structural element, but, using a reaction-diffusion system, it arises quite naturally. In contrast, if the number of structures is quite stable, we have to couple the reaction-diffusion system with other mechanisms to explain the stability. The reliability of the pattern formation mechanism was first discussed by Bard and Lauder (1974) and a possible scenario for robust pattern formation is proposed by Crampin et al. (1999). We introduce the latter in the following subsection.

\section{Growing domain}

In actual biological systems (especially embryonic tissue), the size and shape of the pattern formation field is usually not constant, but grows. The Turing reaction-diffusion system has the property that it retains the periodic structure of fixed wavelength, so if the tissue grows we can expect that the pattern will change according to the growth. This can be illustrated in Fig. 16, where additional peaks are inserted between the originally formed periodic peaks to keep the wavelength fixed (Fig. 16a; we call the white part a 'peak'). 

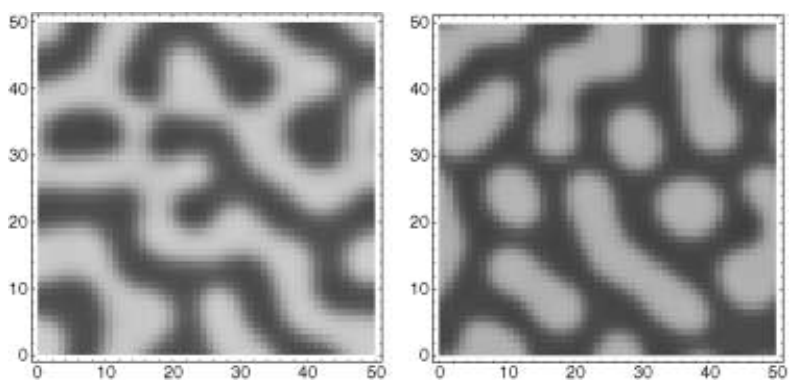

Figure 17. Numerical simulations of the reaction-diffusion model (equations 22) in two spatial dimensions.

Next, we slightly modify the reaction term of the system (Fig. 16b). Note the number of peaks increases as in the previous simulation, but the way they increase is different. In the previous simulation, additional peaks are inserted between pre-existing peaks, but this time each peak is split into two peaks. The type of transition is dependent on the form of the reaction terms. This distinction of peak increase (called 'mode doubling') is investigated analytically by Crampin et al. (2002).

\section{Two dimensions: stripe-spot selection}

We can easily modify the above program for simulation on a two-dimensional spatial domain (a flat surface). In this case, one finds stripes, spots or more complex patterns. For example, we can see, in Fig. 17, a stripe- and spot-like pattern. Stripe-spot selection has been studied in a special case (Ermentrout, 1991; Lyons \& Harrison, 1992), but the mechanism, in general, is not fully understood.

Numerical calculations on two-dimensional curved surfaces (Varea et al., 1999) and three dimensions (Leppänen et al., 2002) have been done recently and some interesting features are observed concerning the connectivity of the periodic pattern.

\section{Future prospects}

As a closing remark, we describe the current status and some future prospects of the application of mathematical models to developmental biology, especially in the pattern formation field.

First, developmental biology itself is still booming by using molecular genetics technology. However, there are several groups of researchers who are trying to understand biological pattern formation using mathematical models. The largest among these groups is that of mathematical biologists, whose background is applied mathematics. The field is expanding rapidly and a considerable number of researchers (although much less than in developmental biology) is involved. This academic area deals with biological phenomena as a whole and includes ecology, population biology and electrophysiology, so only some of the researchers are involved in pattern formation. Another group is that of theoretical physicists, who are doing research on pattern formation in physical phenomena and see biological systems as a subject of application of their theory. There are also sporadic 'mutant' people, who have various backgrounds but are somehow interested in theoretical models of pattern formation. In summary, there is no established course to study pattern formation during development and the number of researchers who take this approach is quite limited.

There exists a huge cultural gap between developmental biologists and theoretical biologists. First, the vocabulary is quite different and these two groups speak 'different languages'. For example, if you put up the equation ' $\partial u / \partial t=f(u, v)+\Delta u$ ' on slides for developmental biologists, approximately $20 \%$ of the audience falls asleep instantaneously. (This is not meant to insult experimental people. Putting the names of more than four molecules in a presentation slide for theoretical people results in a quite similar phenomenon.) In both cases, the actual content is straightforward to understand with proper introduction, but this is difficult to find currently.

The next difference is more fundamental. The goal of research is quite different for experimentalists and theoreticians. In some cases, theoreticians construct models without taking care to explore their biological plausibility. For example, one of the authors (T.M.) has performed some modelling work on certain pattern formation phenomena during development and undertook some experiments to specify which molecule corresponds to which factor in the theoretical model (Miura \& Shiota, 2002). However, when he presented the data, a theoretical physicist asked, 'Judging from the morphological pattern, it's reactiondiffusion. Self-evident. Why do you bother looking for an actual molecule like an FGF?'.

It seems that the experimental application of mathematical models to biological pattern formation is quite a promising area because the lack of progress is more or less due to technical reasons described above, not the lack of importance. There are already plenty of active mathematical biologists working on this topic, which proves the importance of the field. However, there are very few experimental groups who try to apply mathematical models to actual biological experimental systems. As far as we know, there are only two to three groups in the world that can deal with this kind of problem both theoretically and experimentally. Why? It seems that the two cultural differences described above can be an energy barrier for experimental people to enter this field. We hope this text contributes to lowering the first barrier. 


\section{Acknowledgments}

The authors thank Professor Stuart Newman (New York Medical College) and Dr Chad Perlin (Department of Human Anatomy and Genetics, University of Oxford) for their critical reading of the manuscript. This work was supported by the Japan Society for the Promotion of Science.

\section{References}

Asai R, Taguchi E, Kume Y, Saito M, Kondo S (1999) Zebrafish leopard gene as a component of the putative reactiondiffusion system. Mech Dev 89 (1-2), 87-92.

Bard JB (1981) A model for generating aspects of zebra and other mam-malian coat patterns. J Theor Bio/ 93, 363-85.

Bard J (1990) Morphogenesis. Cambridge University Press, Cambridge.

Bard J, Lauder I (1974) How well does Turing's theory of morphogenesis work? J Theor Biol 45, 501-31.

Crampin EJ, Gaffney EA, Maini PK (1999) Reaction and diffusion on growing domains: Scenarios for robust pattern formation. Bull Math Biol 61, 1093-120.

Crampin EJ, Gaffney EA, Maini PK (2002) Mode-doubling and tripling in reaction-diffusion patterns on growing domains: $A$ piecewise linear model. J Math Biol 44, 107-28.

Dowine SA, Newman SA (1994) Morphogenetic differences between fore and hind limb precartilage mesenchyme: Relation to mechanisms of skeletal pattern formation. Dev Biol 162, 195-208.

Downie SA, Newman SA (1995) Different roles of fibronectin in the generation of fore and hind limb precartilage condensations. Dev Biol 172, 519-30.

Ermentrout B (1991) Stripes or spots? Nonlinear effects in bifurcation of reaction-diffusion equations on the square. Proc $R$ Soc Lond A 434, 413-17.

Gilbert SF (2000) Developmental Biology. Sinauer, Massachusettes.

Jiang TX, Jung HS, Widelitz RB, Chuong CM (1999) Selforganization of periodic patterns by dissociated feather mesenchymal cells and the regulation of size, number and spacing of primordia. Development 126, 4997-5009.

Jung HS, Francis-West PH, Widelitz RB et al. (1998) Local inhibitory action of bmps and their relationships with activators in feather formation: Implications for periodic patterning. Dev Biol 196, 11-23.

Kondo S (2002) The reaction-diffusion system: A mechanism for autonomous pattern formation in the animal skin. Genes Cells 7, 535-41.

Kondo S, Asai R (1995) A reaction-diffusion wave on the skin of the marine angelfish pomacanthus. Nature 376, 765-8.
Leppänen T, Karttunen M, Kaski K, Barrio RA, Zhang L (2002) A new dimension to Turing patterns. Physica D 168-9, 3444.

Lyons MJ, Harrison LG (1992) Stripe selection: An intrinsic property of some pattern-forming models with nonlinear dynamics. Dev Dyn 195, 201-15.

Maini PK, Solursh M (1991) Cellular mechanisms of pattern formation in the developing limb. Int Rev Cytol 129, 91133.

Meinhardt H (1995) The Algorythmic Beauty of Sea Shells. Springer-Verlag, Berlin.

Miura T, Maini PK (2004) Speed of pattern appearance in reaction-diffusion models: Implications in the pattern formation of limb bud mesenchyme cells. Bull Math Biol (in press).

Miura T, Shiota K (2000a) Extracellular matrix environment influences chondrogenic pattern formation in limb bud micromass culture: Experimental verification of theoretical models. Anat Rec 258, 100-7.

Miura T, Shiota K (2000b) Tgf $\beta 2$ acts as an 'activator' molecule in reaction-diffusion model and is involved in cell sorting phenomenon in mouse limb micromass culture. Dev Dyn 217, 241-9.

Miura T, Shiota K (2002) Depletion of fgf acts as a lateral inhibitory factor in lung branching morphogenesis in vitro. Mech Dev 116, 29-38.

Miura T, Komori M, Shiota K (2000) A novel method for analysis of the periodicity of chondrogenic patterns in limb bud cell culture: Correlation of in vitro pattern formation with theoretical models. Anat Embryol 201, 419-28.

Moftah MZ, Downie SA, Bronstein NB et al. (2002) Ectodermal fgfs induce perinodular inhibition of limb chondrogenesis in vitro and in vivo via fgf receptor 2. Dev Biol 249, 27082.

Murray JD (2003) Mathematical Biology, 3rd edn. SpringerVerlag, Berlin.

Newman SA, Frisch HL (1979) Dynamics of skeletal pattern formation in developing chicklimb. Science 205, 662-8.

Ros MA, Lyons GE, Mackem S, Fallon JF (1994) Recombinant limbs as a model to study homeobox gene regulation during limb development. Dev Biol 166, 59-72.

Salazar-Ciudad I, Jernvall J (2002) A gene network model accounting for development and evolution of mammalian teeth. Proc Natl Acad Sci USA 99, 8116-20.

Shoji H, Mochizuki A, Iwasa $Y$ et al. (2003) Origin of directionality in the fish stripe pattern. Dev Dyn 226, 627-33.

Turing AM (1952) The chemical basis of morphogenesis. Philos Trans $R$ Soc B 237, 37-72.

Varea C, Aragon JL, Barrio RA (1999) Turing patterns on a sphere. Phys Rev E Stat Physics Plasmas Fluids Relat Interdiscip Top 60, 4588-92.

Wolpert L (1998) Principles of Development. Oxford University Press, Oxford. 


\section{Appendix I}

Table of variables used in the present review.

\begin{tabular}{ll}
\hline Variable & Meaning \\
\hline$x$ & Horizontal length \\
$y$ & Vertical length \\
$d x$ & Horizontal length of small tissue elements \\
$d y$ & Vertical length of small tissue elements \\
$t$ & Time \\
$d t$ & Small interval of time used for numerical calculation \\
$p(n, m)$ or $p$ & Concentration of activator molecule at time $m \times d t$ in $n$th tissue element \\
$q(n, m)$ or $q$ & Concentration of inhibitor molecule at time $m \times d t$ in $n$th tissue element \\
$m$ & Integer number that represents the horizontal position of the tissue \\
$n$ & Integer number that represents time \\
$u(x, t)$ or $u$ & Concentration of activator molecule at time $t$ in position $x$ \\
$v(x, t)$ or $v$ & Concentration of inhibitor molecule at time $t$ in position $x$ \\
$f(p, q)$ or $f(u, v)$ & Function that determines the rate of activator concentration change \\
$g(p, q)$ or $g(u, v)$ & Function that determines the rate of inhibitor concentration change \\
$d_{p}$ & Diffusion coefficient of the activator \\
$d_{q}$ & Diffusion coefficient of the inhibitor \\
\hline
\end{tabular}

